School were also able to see two films, the first dealing with the problem and treatment of drunkenness in Switzerland, and the second with the utilization of fruit, either in a fresh or preserved state, for human consumption or as a source of unfermented juices which can be made into most attractive drinks the popularity of which is increasing year by year.

R. WEATHERALL

\section{CO-OPERATION IN BOTANICAL RESEARCH}

$F^{\circ}$ OR his presidential address to the Botanical Society of America (December 28, 1946), Dr. I. W. Bailey, chairman of the Institute for Research in Plant Morphology at Harvard University, selected the theme of "Co-operation Versus Isolation in Botanical Research" (Chronica Botanica, 11, 1947).

In a world subject to profound and drastic changes, Dr. Bailey considers it inevitable that botanical science must also be affected, particularly the older branches of the subject. To quote his own words, he raises "the challenging question, what roles should taxonomy, anatomy, palæobotany and other phases of morphology be expected to play in future developments ?". A whole book could be devoted to the answer. Certainly the way has been prepared for a brisk and lively discussion.

"There are, of course, two extreme views regarding the matter. On the one hand, there are individuals who believe that these largely descriptive disciplines are moribund, if not actually over-ripe for interment, and that funds now dissipated for their support should be diverted into productive ultra-modern, experimental channels. On the other hand, there are those laissez-faire individuals who naively assume that civilization must continue to support their endeavours regardless of how unproductive their viewpoints and methodologies may have become.

"The latter individuals are living in a dream world of their own creation which is destined sooner or later to be rudely shattered. The former individuals fail to visualize the extreme complexity and variability of biological phenomena, and thus overlook or ignoro numerous significant morphological variables in their experimental material."

In the first place, he asks if the extensive morphological researches of the last hundred years have not provided sufficient data to serve the purposes of contemporary and future experimental disciplines, in particular, those of the physiologist, the biochemist and the biophysicist. When these workers turn to the standard texts, can they find in them the information which they require? Dr. Bailey replies in the negative, and in this he will be supported by most botanists who have given thought to the matter. Many of the supposedly fundamental ideas of plant anatomy are inadequate, based as they were on researches undertaken in relation to phylogenetic questions. An impartial consideration of almost any of the major problems of contemporary interest will reveal how much scope still remains for morphological inquiry. To quote Dr. Bailey : "It is evident that significant progress in the solution of important botanical problems is being retarded by inadequacies of existing morphological data and by the unquestioning acceptance of unreliable generalizations". He then asks how these difficulties are to be avoided in the future, and emphasizes the dangers and draw. backs of premature specialization in instruction and research. "This encystment of botany in specialized subdivisions encourages premature and excessive generalizations from limited induction and tends to inhibit unified attacks along broad fronts, such as are essential for the solution of the more complex and difficult types of botanical problems." The solution of any of the major problems of biology requires the close co-operation of workers in different fields and a harmonizing of the data of the several contributory disciplines. Various means are available whereby co-operation can be encouraged, but as to how the latter aim is to be achieved Dr. Bailey is rather less specific. The magnitude of the task is oppressive : in practice it will call for the sustained activities of a team of workers. Yet, as experience teaches, major biological syntheses depend on the special qualities and capacities of particular individuals, and these, apparently, are of rare and sporadic occurrence.

Morphologists, whether adherents of old or new conceptions, are not alone in being scourged by Dr. Bailey. "If one probes deeply into the applications of physics and chemistry in the analysis of plant materials one likewise finds evidences of premature and excessive generalization from restricted viewpoints. I have encountered this tendency in exaggerated form in chemical analysis of plant tissues and particularly in physical and chemical investigations of the walls of plant cells. The specialist in specific physical and chemical methodologies is likely to run astray unless his deductions are verified by individuals who are thoroughly familiar with the full complex of structural variables in a wide range of plants."

But Dr. Bailey is by no means pessimistic in his outlook for plant morphology. He views with confidence a future in which taxonomy, anatomy and other morphological disciplines will be stimulated and broadened by close contact with one another and with other branches of the science. The result should be a closer approach to the solution of the central problems of biology. "To facilitate such mutually beneficial relationships, it is essential to break down existing emotional and intellectual barriers." The morphologist must recognize the limitations of his own techniques and appreciate the assistance which other disciplines can afford him in solving problems in his own particular field. "He must acquire sufficient familiarity with chemistry and physics to enable him to talk intelligently with workers in other disciplines." In emphasizing this point, Dr. Bailey's observations are no doubt pertinent in relation to some of the research institutions in the United States : in a majority of British universities, a reasonable knowledge of chemistry and physios is now regarded as an essential preparation for botanical studies.

A particular interest is attached to that section of the address in which Dr. Bailey attempts to show how co-operation between the different subdivisions of botany is to be achieved. "It cannot be coerced or developed directly by administrative fiat. It must arise naturally and more or less spontaneously or not at all. The wisest and most effective procedure should be to create a general environment that encourages rather than inhibits co-operative efforts." To some extent this can be achieved by wise administration, particularly by making suitable appointments and the judicious allocation of funds. An allocation of funds which tends to encourage excessive diversification and specialization and which favours 
"unbridled individualism" is to be deplored. "Such policies may yield spectacular and highly significant results during the pioneering stages of a new trend of specialization, but frequently leave a large residue of injurious consequences, and do not create a general environment favourable for co-operative endeavours. I do not imply by this that one should make a fetish of team-play or that the lone wolf and the prima donna should be eliminated and replaced by amiable mediocrities, but rather that the training of graduate students should be broadened and that where individuals of recognized ability wish to co-operate in research, funds to encourage such relationships should be available. . . . We must now strive in botany as in national affairs to prevent a reversion to isolation and to intellectual encystment."

Even in the larger universities in the United States it is not anticipated that extensive schools of research in all the subdivisions of botany can be supported. Dr. Bailey advocates that each university department should develop strength in particular directions and that students should be free to move from one university or institution to another according to their special interests.

In concluding, Dr. Bailey considers how the funds available for botanical research can best be used. One of the methods commonly adopted is to give handsome support to one or two leading individuals who have established a reputation in a particular field. This he regards as possibly the easiest and least expensive way for some institutions; it may even be the easiest and least expensive way of advancing the frontiers of science. But other possibilities should not be overlooked. Policies of the kind mentioned above commonly lead to narrow specialization and they "tend to inhibit attacks upon major biological problems which require the concatenated efforts of different disciplines for their solution". In their more exaggerated forms, they may in time create such a complex of competitive subdivisions as to render impossible the integration of the data of the several branches. Botanical science must inevitably suffer from the attendant disruption.

C. W. WARDLAW

\section{FORMULA AND BRAIN WEIGHTS}

DROF. E. W. COUNT ${ }^{1}$ has recently examined the Iarious formulæ which have been suggested for expressing the relationship between brain-weight and body-weight in vertebrates, and has also introduced a new formula. In his extensive study he states that his objects are: "(I) to formulate the growth behaviour between brain and body weights in human ontogeny, (2) to find any traits in common with other Primates and Mammalia, (3) to formulate analogous behaviour in comparative anatomy; and (4) to search for any connexions between ontogeny and comparative anatomy". His paper is divided into five sections : (1) is introductory, discussing method and terminology; (2) deals with the data and formulæ from the point of view of ontogeny; (3) discusses the comparative anatomy problem, includes an extensive criticism of previous work and introduces the author's new formula; (4) attempts to relate the ontogenetic results to the phylogenetic; and (5) is a summary. The paper concludes with an extensive bibliography and a number of tables of data.
If we denote brain-weight by $y$, body-weight by $x$, and parameters by $a, b, c$, Prof. Count's main conclusions appear to be as follows:

(a) Ontogenetically, in both fœetal and postinfantile stages, the relationship $y=a x^{b}$ will hold.

(b) Phylogenetically, for mammals the relationship is, $y=a e^{b x-c x 2}$; for reptiles $y=a e^{b x+c x^{2}}$. The form $\log y=\log a+b \log x \pm c(\log x)^{2}$ is termed the 'cephalization function'.

(c) If the intersection of the reptilian cephalization exponent with the general mammalian exponent is taken as a new origin, then the mammalian exponent may be considered as a transformation of the reptilian.

(d) Increase in absolute body-size is requisite for higher cephalization. This latter term is nowhere clearly defined, but appears to be related to some concept of more complicated structure and function.

Prof. Count's discussion of the data and their sources is full and most welcome; he not only supplies the reader with lists of some of the available data, but also includes tables in which these data are collected. Unfortunately, many of these figures are mean-values and consequently of considerably less value than the individual observations. For some reason Dr. H. B. Latimer's excellent data on cats are not included, and are considered to be "too good"; no reason for such a rejection is given.

Snell ${ }^{2}$ was first to suggest the use of the formula $y=a x^{b}$, and the adoption and development of this formula for brain/body weights by Dubois, Lapicque and Brummelkamp is well known. These authors have maintained that the value of the parameter $b$ is 0.56 for different but related species of vertebrates, but is $\mathbf{0 . 2 6}$ for animals of different sizes but belonging to the same species. Brummelkamp, moreover, has stated that the values of $\log a$ are always integral multiples of $\sqrt{ } 2$. Count discusses this work in considerable detail and rightly concludes that these suggested values for the parameters have no adequate foundation. This work has already been criticized by v. Bonin, who has treated the data from a different point of view and fits a straight line to the logarithms of the body-weights and brain-weights of mammals, concluding that the value of $b$ was 0.655 while the value of $a$ varies from species to species. Count's objection to v. Bonin's treatment seems to be mainly subjective; he thinks that mammals form too large a group, and considers that if two animals have the same body-weight, the one with the larger brain will be the more intelligent. The method of v. Bonin does not lead to this conclusion.

Many of these criticisms are well founded, especially those directed against the Dubois-LapicqueBrummelkamp school, but other equally radical criticisms may be made which would also apply to Count's own treatment-the mathematical treatment is quite inadequate.

If it is decided to fit a curve $y=f(x, a, b, c \ldots)$, where $x$ is the independent variable and $a, b, c$ are parameters, it is necessary to make certain assumptions about the distribution of the variables. If we fit by the method of least squares, we usually assume that the $x$ 's are free from error and the $y$ 's are norm. ally distributed; the values of the parameters and their standard errors can then often be estimated. The authors discussed by Count, and Count himself, assume that the results of fitting

$$
y=a x^{b} \text { and } \log y=\log a+b \log x
$$

will be the same. This is not the case, since we assume $y$ to be normally distributed with $x$ free 